\title{
Asymptomatic double subclavian "steal" phenomenon: case report
}

\begin{abstract}
Significant stenosis or occlusion of subclavian artery proximal to the vertebral artery origin due to atherosclerotic vascular disease causes the reversal blood flow in vertebral artery called the subclavian "steal" syndrome. It often remains as an asymptomatic phenomenon if the circulation in ipsilateral arm is sufficient and reversal flow do not cause the significant steal of blood from circle of Willis arteries. We describe the case of asymptomatic double steal phenomenon caused by multiple atherosclerotic stenoses in both subclavian and brachiocephalic arteries. Examination of extracranial and brain arteries by ultrasound methods and computed tomography angiography helped to determine the degree of blood deficit in each basin and to apprise the sufficiency of compensation.
\end{abstract}

Keywords: subclavian steal syndrome, subclavian steal phenomenon, stenosis of brachiocephalic artery, occlusion of subclavian artery, retrograde flow, reversive flow, circle of Willis arteries, cerebral collateral circulation, cerebral circulation, colorcoded duplex ultrasonography of craniocervical arteries, transcranial color-coded duplex ultrasonography, computed tomography angiography
Volume 9 Issue I - 2019

\author{
Galina Baltgaile,' Galina Antonuk, ${ }^{2}$ Helmuts \\ Kidikas $^{3}$ \\ 'Department of Neurology, Med. Clinic ARS, Riga, Latvia \\ 'Departmnet of Ultrasonography, Association of Health Centres, \\ Riga, Latvia \\ ${ }^{3}$ Department of Radiology, Med. Clinic ARS, Riga, Latvia
}

Correspondence: Galina Baltgaile MD, Neurologist, Department of Neurology, Medical Clinic ARS, Skolas 5, LV-I0I0, Riga, Latvia, Email baltgaile@gmail.com

Received: March 18, 2018 | Published: February 08, 2019
Abbreviations: VA, vertebral artery; SA, subclavian artery; $\mathrm{BCA}$, brachiocephalic artery; CA, carotid artery; CCDS, color-coded duplex ultrasonography; TCCS, transcranial color-coded duplex ultrasonography; CTA, computed tomography angiography

\section{Introduction}

Subclavian steal phenomenon occurs when a SA stenosis or occlusion proximal to the VA origin causes retrograde flow in the ipsilateral vertebral artery. Most literatures report the prevalence of subclavian steal syndrome as between $0.6 \%$ to $6.4 \% .^{1-3}$ The syndrome is often asymptomatic The Joint Study of Extracranial Arterial Occlusion by Fields et al. ${ }^{4}$ yielded $2.5 \%$ incidence (168/6534), with only $5.3 \%$ of these patients experiencing neurological symptoms. ${ }^{4}$

Some authors have suggested that the patients who develop symptoms from this phenomenon usually have additional vascular pathology involving either the intracranial or extracranial vessels. ${ }^{1,5}$ The presence of clinical symptoms depends on sufficiency of circulation in the ipsilateral arm and degree of "stolen" blood flow from the contralateral vertebral artery and from arteries of circle of Willis. Appliance of non-invasive ultrasound methods and CTA for examination of brain arteries can help to reveal not only the arterial stenotic lesions but also the direction and parameters of flow in a cases of "steal" syndrome.

\section{Case presentation}

Male patient 67 y.o. with no complaints or signs of disease has been referred to the stroke prevention centre for preventive ultrasound examination of blood vessels because of hyperlipidaemia and heavy smoking for more than 20 years. Patient had no any cardiac or neurologic symptoms. Blood pressure in a both arms was symmetrically low $-90 / 40 \mathrm{~mm} . \mathrm{Hg}$.

During the examination of craniocervical blood vessels by CCDS and brain blood vessels by TCCD multiple sclerotic lesions of arteries with subclavian steal phenomenon in both sides has been determined. CTA confirmed all the stenoses detected by ultrasound. The correlation of accuracy of the degree of stenosis more that $70 \%$ between two methods was very high. Detection of the stenotic degree less than $60 \%$ was different by two applied methods because of known difference of calculation by ESCT method (mainly applied in CCDS) and NASCET method (applied in CTA).

\section{Results of examination}

Patient had left SA completely occluded with the retrograde flow via left VA- left subclavian "steal" syndrome. Normal velocity retrograde flow in $\mathrm{V}_{0}-\mathrm{V}_{2}$ segments of left VA became reversal anteretrograde in Atlas segment and changed the direction to anterograde in intracranial part because of collateral supply (Figure 1) (Figure 2).

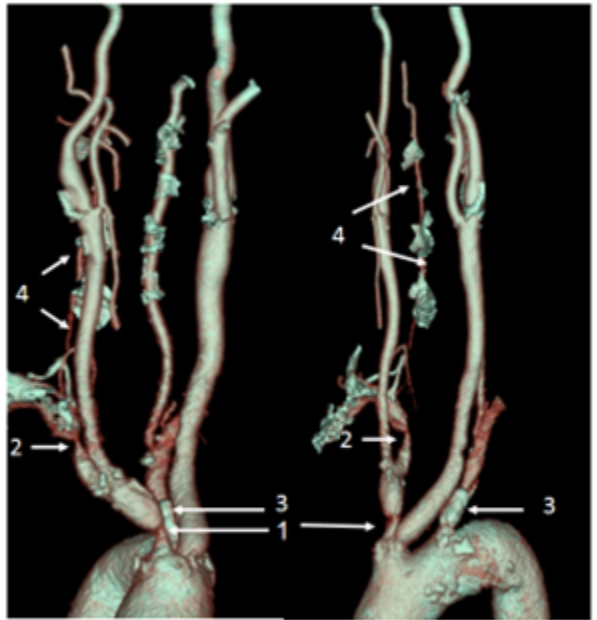

Figure I CTA with 3D reconstruction of craniocervical arteries ( with rotation on the left side): I $-90 \%$ stenosis of BCA, $2-70 \%$ stenosis of right $\mathrm{SA}$, 3- occlusion of left SA, 4- hypoplastic rightVA.

High flow velocity was found at the origin of the left CA insignificantly narrowed by atherosclerotic plaques on $30-50 \%$. This discrepancy between high flow velocity and middle grade of stenosis can be explained by redistribution of flow due to occlusion of SA.

BCA was narrowed by $90 \%$ with high turbulent flow at the site of stenosis and characteristic drop of flow in distal parts. Blood flow in 
the right $\mathrm{CA}$ and the right SA had low velocity with a typical poststenotic flow's spectrum. Right SA was narrowed by $70 \%$ (Figure 1). Significant drop of flow distally to stenosis partly was compensated by retrograde flow via right VA- defined as a right subclavian "steal" syndrome. Low velocity retrograde flow was detected in all parts of hypoplastic right VA. Two stenoses were detected in the right CA: at the origin and in bifurcation. 30\% degree stenosis (by NASCET) and $40 \%$ degree stenosis (by ESCT) at the origin and $30 \%$ stenosis (by NASCET) in the bifurcation did not change significantly flow velocity, which remained very low from the beginning (Figure 1) (Figure 3).

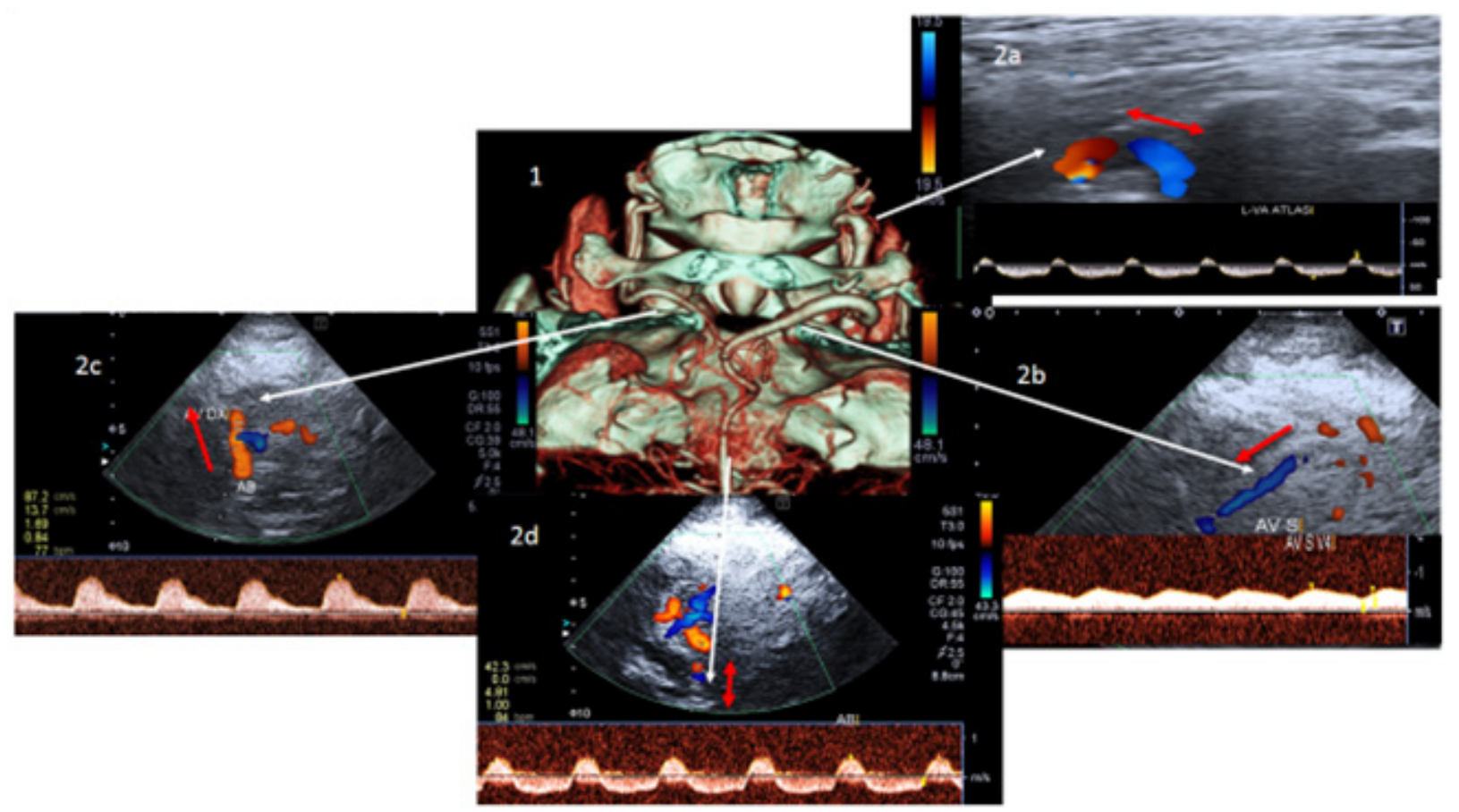

Figure 21 - CTA with 3D reconstruction of arteries on the basis cranii; 2- CCDS(a) and TCCD (b,c,d) with Doppler spectrum of the same arteries: 2a-reversive ante-retrograde flow in the Atlas segment of the left VA; $2 \mathrm{~b}$ - anterograde flow in the intracranial part of the left VA; 2c- retrograde flow in intracranial part of the right VA; $2 \mathrm{~d}$-reversive ante-retrograde flow in the basilar artery; (flow direction is marked with the arrows)

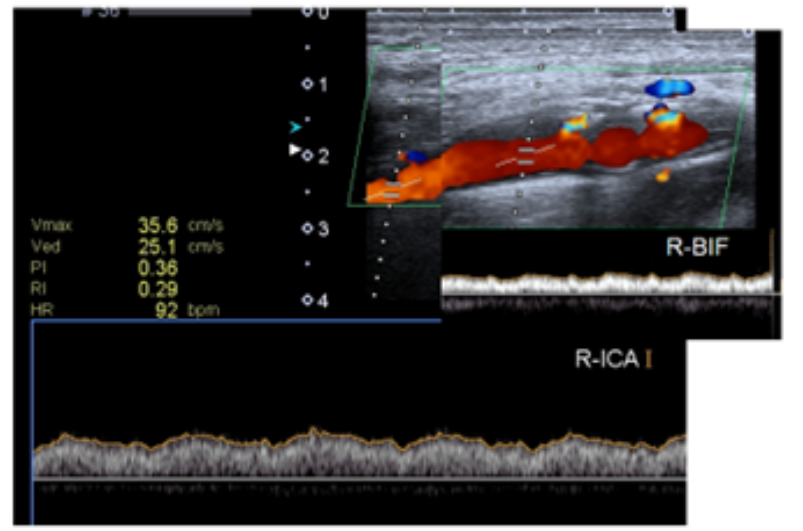

Figure 3 CCDS with Doppler spectrum of right CA in the bifurcation (right side) and the proximal part of right internal CA (left side). Note the very low velocity flow with changed Doppler post-stenotic spectrum.

TCCD revealed low velocity flow with changed Doppler spectrum in the right middle cerebral artery, compensated by collateral flow via anterior communicating artery and right posterior communicating artery (Figure 4). Flow velocity was normal in the left anterior and middle cerebral arteries. Working left posterior communicating artery served as a collateral pathway to the left posterior artery. Flow in the left vertebral artery changed its direction from the reversive anteretrograde flow in Atlas segment to the normal direction in intracranial part while right vertebral artery "stole" blood by retrograde flow (Figure 2). Flow in a basilar artery remained reversive ante-retrograde in all segments as well as at the origin of both posterior arteries.

After ultrasound and CTA examinations it was concluded that decreased flow's velocity and pressure in both SA and BCA was not enough compensated by retrograde flow via both VA. Decreased flow in the right carotid and middle cerebral arteries caused by stenosis of BCA was just partly compensated via collaterals of circle of Willis. Practically only left internal CA and collateral supply to the intracranial part of left VA were main sources for the compensation of cerebral circulation.

Despite of asymptomatic course of disease and the absence of symptoms at the moment of observation it was decided to place stents in the BCA and right SA. It was suggested to assess circulation in neck's and brain arteries after restoration of normal flow in the right CA, SA and VA and to decide about the necessity of the left carotidsubclavian shunt placement.

\section{Discussion}

Many authors assume that subclavian steal phenomenon is a benign condition since the reversed blood flow in the ipsilateral vertebral artery often serves as an effective collateral pathway for the affected arm. ${ }^{6}$ Significant ischemia of the arm is therefore rare, even in patients who have complete occlusion of the proximal SA., 


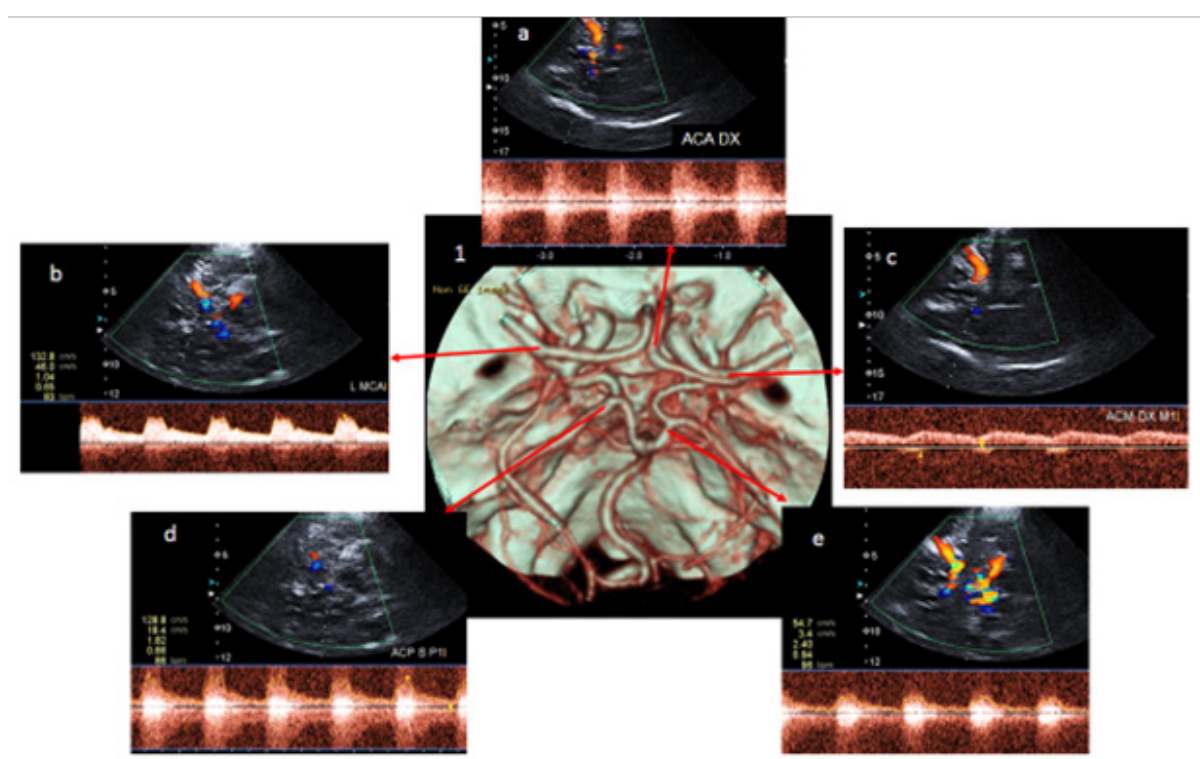

Figure 4 1- CTA with 3D reconstruction of circle of Willis arteries; a,b,c,d,e - TCCD with Doppler spectrum of the same arteries: a- high velocity flow in the right anterior cerebral artery through the anterior communicating artery; b- low velocity and changed spectrum flow in the right middle cerebral artery; d- high velocity flow to the left posterior cerebral artery via left posterior communicating artery; e- blood flow in the right posterior artery.

In our case significant insufficiency of blood flow in both SA caused by occluded SA on one side and significantly narrowed BCA and SA on other side was not completely compensated by retrograde flow in VA and blood pressure in both arms was low. Despite of this patient had no signs of vascular insufficiency in arms. The most of steal phenomenon cases are vertebro-vertebral steal type, when blood is stolen from one VA to the opposite via basilar artery. In such cases blood flow in the middle and distal part of basilar artery and other circle of Willis arteries stay untouched, which explains the absence of clinical symptoms. Common symptom is dizziness exacerbated by intensive work by hands. The most of patients who suffered from chronic posterior circulation ischemia due to subclavian steal phenomenon had additional vascular lesions in extracranial or intracranial vessels. ${ }^{5,8}$ It seems quite unusual that in our case of multiple stenoses and occlusion of craniocervical arteries with reversed flow in both VA no signs of insufficiency in posterior cerebral circulation appeared. Even intensive work by hands did not provoke dizziness. While CTA revealed working anterior and both posterior communicating arteries, TCCD helped to define the direction of flow in circle of Willis arteries. Flow in the right middle cerebral artery apparently was compensated through communicating arteries. Taking into account the reversal ante-retrograde flow in basilar artery it seems that collateral flow from preserved left internal carotid artery through left posterior communicating artery could compensate posterior circulation.

The absence of clinical symptoms in this case did not prove the adequacy of the physiological compensation of blood flow in the subclavian and cerebral arteries. Bilateral low brachial arterial pressure was the result of the failure of retrograde vertebral "steal". Working communicating cerebral arteries could not ensure the normal distribution of blood in all cerebral basins, since only the blood flow in the left ICA was sufficient. A complete reverse ante-retrograde course in BA rather indicated a compensation threshold.

Judged by how unstable was this compensation the decision about endovascular treatment seems logic even in the absence of symptoms. Stents placement in BCA and right SA helped to restore normal flow in a right ICA, VA and SA thus to prevent the appearance of clinical signs of cerebrovascular insufficiency due to the break of compensation. Endovascular procedure followed by the medication with antiplatelet and statin prevent relative risk of ischemic stroke by elimination of low-flow and thromboembolism from proximal plaques.

\section{Conclusion}

Examination of extracranial and intracranial vessels by ultrasound was very sensitive to determine multiple stenoses of arteries and evaluate the circulation in asymptomatic case of double subclavian steal phenomenon. While CTA helped to determine all stenoses and working collateral pathways sometimes poorly visible by ultrasound it did not show the direction and parameters of flow and could not help to evaluate completely the sufficiency of compensation in this particular case. Both methods CTA and TCCD should be recommended for the appraisal of steal phenomenon in cases of multiple stenoses of craniocervical arteries.

\section{Acknowledgments}

None

\section{Conflicts of interest}

The authors declare no conflicts of interest.

\section{References}

1. Osiro S, Anna Zurada A, Gielecki J, et al. A review of subclavian steal syndrome with clinical correlation. Med Sci Monit. 2012;18(5):RA57RA63.

2. Tan TY, Schminke U, Lien LM, et al. Subclavian steal syndrome: can the blood pressure difference between arms predict the severity of steal? $J$ Neuroimaging. 2002;12(2):131-135.

3. Hennerici M, Klemm C, Rautenberg W. The subclavian steal phenomenon: a common vascular disorder with rare neurologic deficits. Neurology. 1988;38(5):669-673.

4. Fields WS, Lemak NA. Joint study of extracranial arterial occlusion. VII. Subclavian steals - a review of 168 cases. JAMA. 1972;222(9):11391143 . 
5. Webster MW, Downs L, Yonas H, et al. The effect of arm exercise on regional cerebral blood flow in the subclavian steal syndrome. Am J Surg. 1994;168(2):91-93.

6. Bron C, Hirt L, Halabi G, et al. Asymptomatic high flow subclavian steal in a patient with hemodialysis access. $J$ Vasc Access. 2010;11(1):63-65.
7. Gosselin C, Walker PM. Subclavian steal syndrome: existence, clinical features, diagnosis and management. Semin Vasc Surg. 1996;9(2):93-97.

8. Sharma V, Chuah $\mathrm{B}$, Teoh $\mathrm{H}$, et al. Chronic brainstem ischemia in subclavian steal syndrome. J Clin Neurosci. 2010;17(10):1339-1341. 